SLAC-PUB-12240

January 2007

(A)

\title{
Start-to-end transport design and multi-particle tracking for the ILC electron source*
}

\author{
F. Zhou, Y. Batygin, A. Brachmann, J. Clendenin, R. H. Miller, J. C. Sheppard, \\ and M. D. Woodley \\ Stanford Linear Accelerator Center, 2575 Sand Hill Road, Menlo Park, \\ CA 94025, USA
}

\begin{abstract}
A train of 2-ns micro bunches of longitudinally polarized electrons are generated in a 120-kV DC-gun based injector in the ILC electron source; a bunching system with extremely high bunching efficiency to compress the micro-bunch down to 20 ps FWHM is designed. Complete optics to transport the electron bunch to the entrance of the $5-\mathrm{GeV}$ damping ring injection line is developed. Start-to-end multi-particle tracking through the beamline is performed including the bunching system, pre-acceleration, chicane, $5-\mathrm{GeV}$ superconducting booster linac, spin rotators and energy compressor. It shows that $94 \%$ of the electrons from the DC-gun are captured within the damping ring 6-D acceptance $A_{x}+A_{y} \leq 0.09 \mathrm{~m}$ and $\Delta E \times \Delta z \leq( \pm 25 \mathrm{MeV}) \times( \pm 3.46 \mathrm{~cm})-$ at the entrance of the damping ring injection line. The field and alignment errors and orbit correction are analyzed.
\end{abstract}

* Work supported by DOE contract No. DE-AC02-76SF00515 


\title{
Start-to-end transport design and multi-particle tracking for the ILC electron source
}

\author{
F. Zhou, Y. Batygin, A. Brachmann, J. Clendenin, R. H. Miller, J. C. Sheppard, \\ and M. D. Woodley \\ Stanford Linear Accelerator Center, 2575 Sand Hill Road, Menlo Park, \\ CA 94025, USA
}

\begin{abstract}
A train of 2-ns micro bunches of longitudinally polarized electrons are generated in a 120-kV DC-gun based injector in the ILC electron source; a bunching system with extremely high bunching efficiency to compress the micro-bunch down to 20 ps FWHM is designed. Complete optics to transport the electron bunch to the entrance of the 5-GeV damping ring injection line is developed. Start-to-end multi-particle tracking through the beamline is performed including the bunching system, pre-acceleration, chicane, 5-GeV superconducting booster linac, spin rotators and energy compressor. It shows that $94 \%$ of the electrons from the DC-gun are captured within the damping ring 6-D acceptance $A_{x}+A_{y} \leq 0.09 \mathrm{~m}$ and $\Delta E \times \Delta z \leq( \pm 25 \mathrm{MeV}) \times( \pm 3.46 \mathrm{~cm})-$ at the entrance of the damping ring injection line. The field and alignment errors and orbit correction are analyzed.
\end{abstract}

\section{Overview}

The ILC electron injector consists of a DC-gun incorporating a semiconductor photocathode illuminated by a Ti:sapphire drive laser to produce a train of 2-ns long micro bunches. A highly efficient bunching system consisting of two subharmonic prebunchers (SHB) and a $\beta$-matched L-band Traveling Wave (TW) buncher compress the bunch length to 20 ps FWHM. The electron bunches are then accelerated to $76 \mathrm{MeV}$ in a normal conducting L-band structure. A chicane downstream of this pre-acceleration system is used to clip off the low energy tail of the bunched beam. After traversing an emittance measurement station downstream of the chicane, the electron bunch is accelerated to $5 \mathrm{GeV}$ using a booster linac consisting of standard ILC-type superconducting (SC) cryo-modules. During transport of the beam from the electron booster linac to the damping ring (DR) injection line, the electron spin vector is adjusted vertical and the energy is compressed. The overall optics and geometry of the electron source are shown in Figs. 1 and 2, respectively.

This technical note is organized as follows: a system description including the bunching system, chicane, booster linac, spin rotators, and energy compressor is presented in section 2; section 3 presents the multi-particle tracking from the gun to the entrance of the damping ring injection line without and with magnet errors and orbit corrections; and, finally, the results are summarized in section 4. 


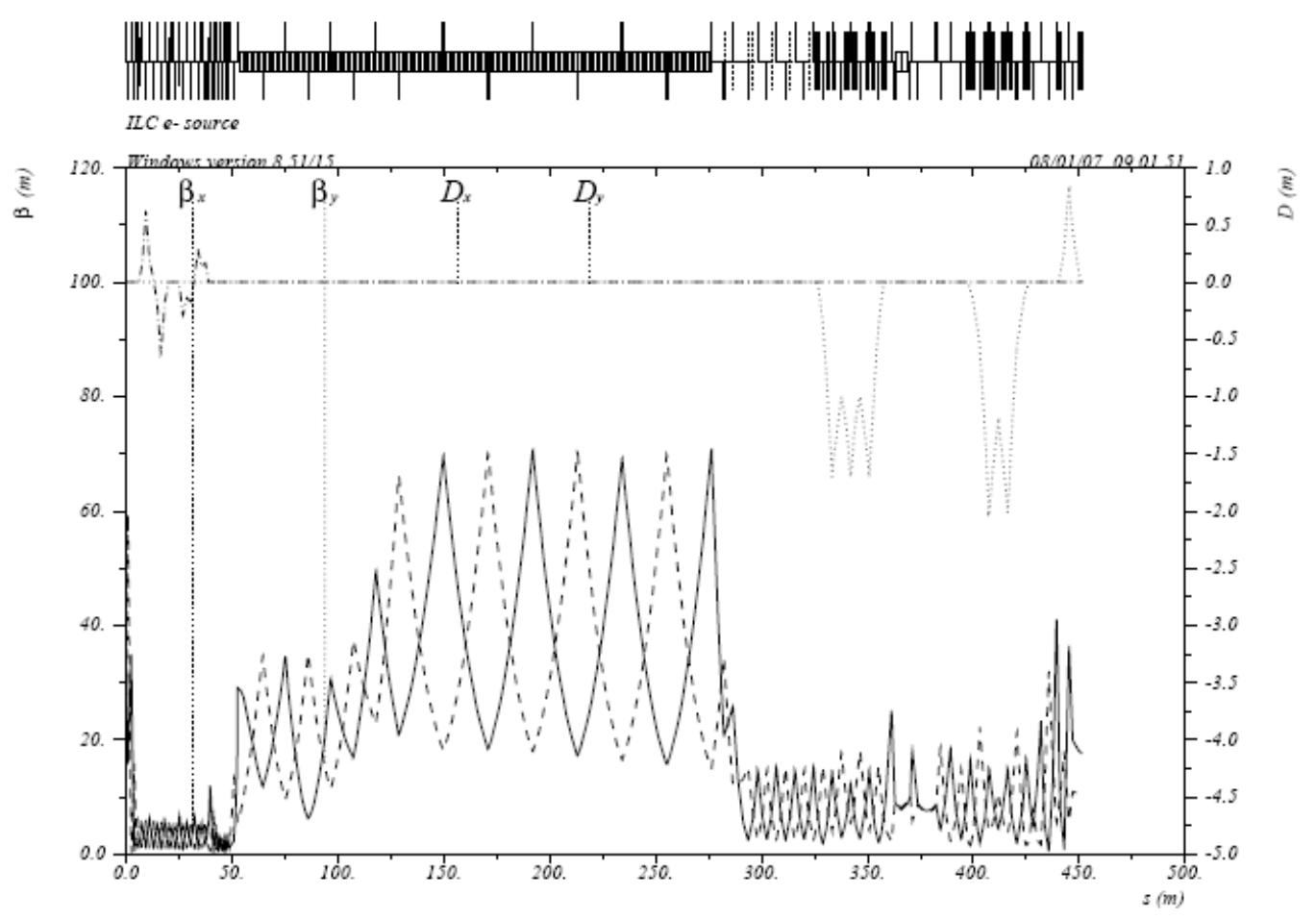

FIG.1. Overall optics of the ILC electron source (76 MeV injector is not shown).

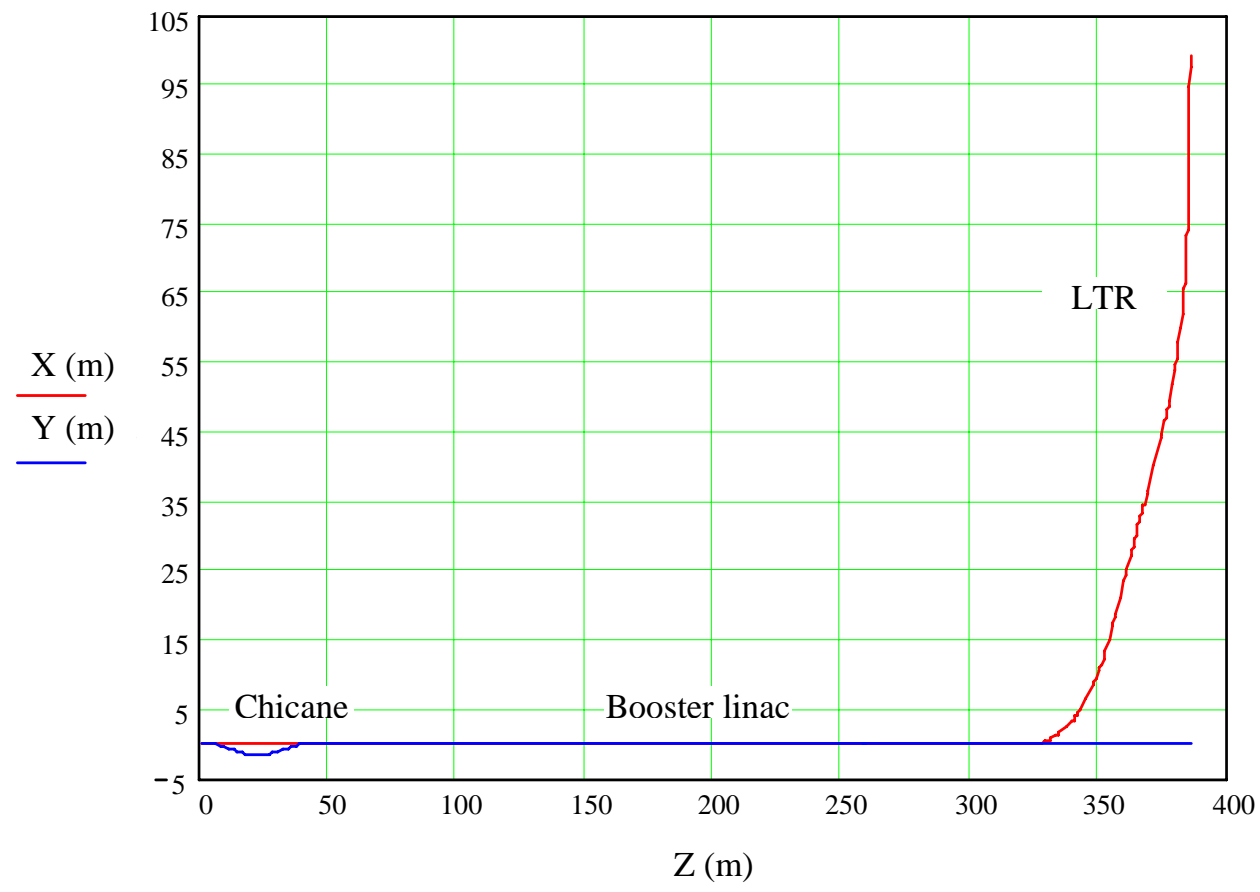

FIG. 2. Overall geometry of the ILC electron source (76 MeV injector is not shown). 


\section{System description}

\subsection{6-MeV injector with high bunching efficiency}

A train of 2-ns micron bunches are generated in a 120-kV DC gun based injector. A bunching system is proposed to compress the micro-bunch down to 20 ps FWHM from 2 ns. It includes two SHBs and an L-band buncher. Two SHBs working at $108 \mathrm{MHz}$ and $433 \mathrm{MHz}$ [1], respectively, are adopted for our design. Each SHB needs $\sim 50 \mathrm{kV}$ of high voltage. The microbunch is first compressed down to 200 -ps FWHM ( $94^{\circ}$ at L-band) by the two SHBs. Further compression down to 20 ps FWHM is achieved by the L-band buncher. In the TESLA Technical Design Report, two 5-cell, $\beta=1$ Standing Wave (SW) structures are used as bunchers [1]. In our design, a 5-cell L-band TW structure with constant $\beta=0.75$ and two 50-cell $\beta=1$ TW normal conducting L-band structures are used as the buncher and pre-acceleration system, respectively.

\subsubsection{Results with $\beta=0.75$ L-band TW buncher}

The schematic layout of the injector with the TW buncher is shown in Fig. 3. The gun is placed at a $20^{\circ}$ angle to the injector axis. This arrangement has some technical advantages: allows the laser beam to access the photocathode; permits polarization measurements; protects the gun vacuum; and allows the installation of a second backup gun. The distance from the gun exit to the bend is $75 \mathrm{~cm}$, which is similar to the SLC injector. The distance between the bend and the first SHB (108 MHz) is $75 \mathrm{~cm}$. The beam transported from the gun to the first SHB is focused by two magnetic lenses so that they can be used to adjust the radius and convergence of the beam at the entrance of the solenoid that confines the beam in the SHBs. The distance between the two SHBs is 202 $\mathrm{cm}$, and the solenoid field in the region ramps from $50 \mathrm{G}$ to $135 \mathrm{G}$. The distance between the second SHB and the entrance of the L-band buncher is $33 \mathrm{~cm}$, which allows the installation of diagnostics. The solenoid field ramps from $135 \mathrm{G}$ in the second SHB to $660 \mathrm{G}$ in the buncher to keep the beam small. While the primary bunching is achieved by the L-band buncher, a final, relatively small, increment of bunching takes place in the first several cells in the pre-accelerator, which immediately follows the L-band buncher. The accelerating gradients in the L-band buncher and pre-accelerator are $5.5 \mathrm{MV} / \mathrm{m}$ and $8.5 \mathrm{MV} / \mathrm{m}$ [2], respectively, and the beam is accelerated to $76 \mathrm{MeV}$ by the end of the injector. The first $\sim 1.5 \mathrm{~m}$ of the L-band sections are immersed in a $660-\mathrm{G}$ solenoid field to focus the beam, and then the field is tapered down to zero as the beam gains energy. Beam modeling using PARMELA [3] is performed starting from the 120-kV DC-gun exit to the 76-MeV injector exit. To account for losses through the injector, a bunch charge of $6.4 \mathrm{nC}$ is assumed at the gun (twice the charge required at the IP). The unnormalized edge emittance at the gun exit is assumed to be $70 \mu \mathrm{m}$. Fig. 4 shows the energy and phase spectra at the gun, L-band buncher and 76-MeV injector exit, respectively. It is shown that at the injector end the energy spread is $<100 \mathrm{keV}$ FWHM ( $<1.5 \mathrm{MeV}$ Full Width) and the bunch length is $9^{\circ}$ at L-band or 20 ps FWHM ( $25^{\circ}$ at L-band or 53 ps Full Width). The beam envelope and solenoid field map along the injector are shown in Figs. 
5 and 6, respectively. The normalized rms emittance vs longitudinal position is shown in Fig. 7.

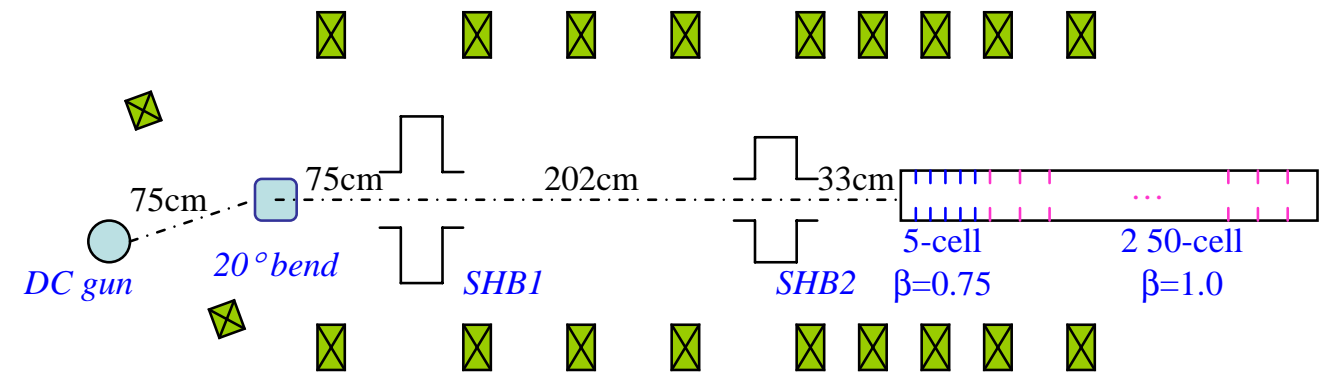

FIG. 3. Schematic layout of the ILC 76-MeV electron injector; dimensions are not scaled in the plot.
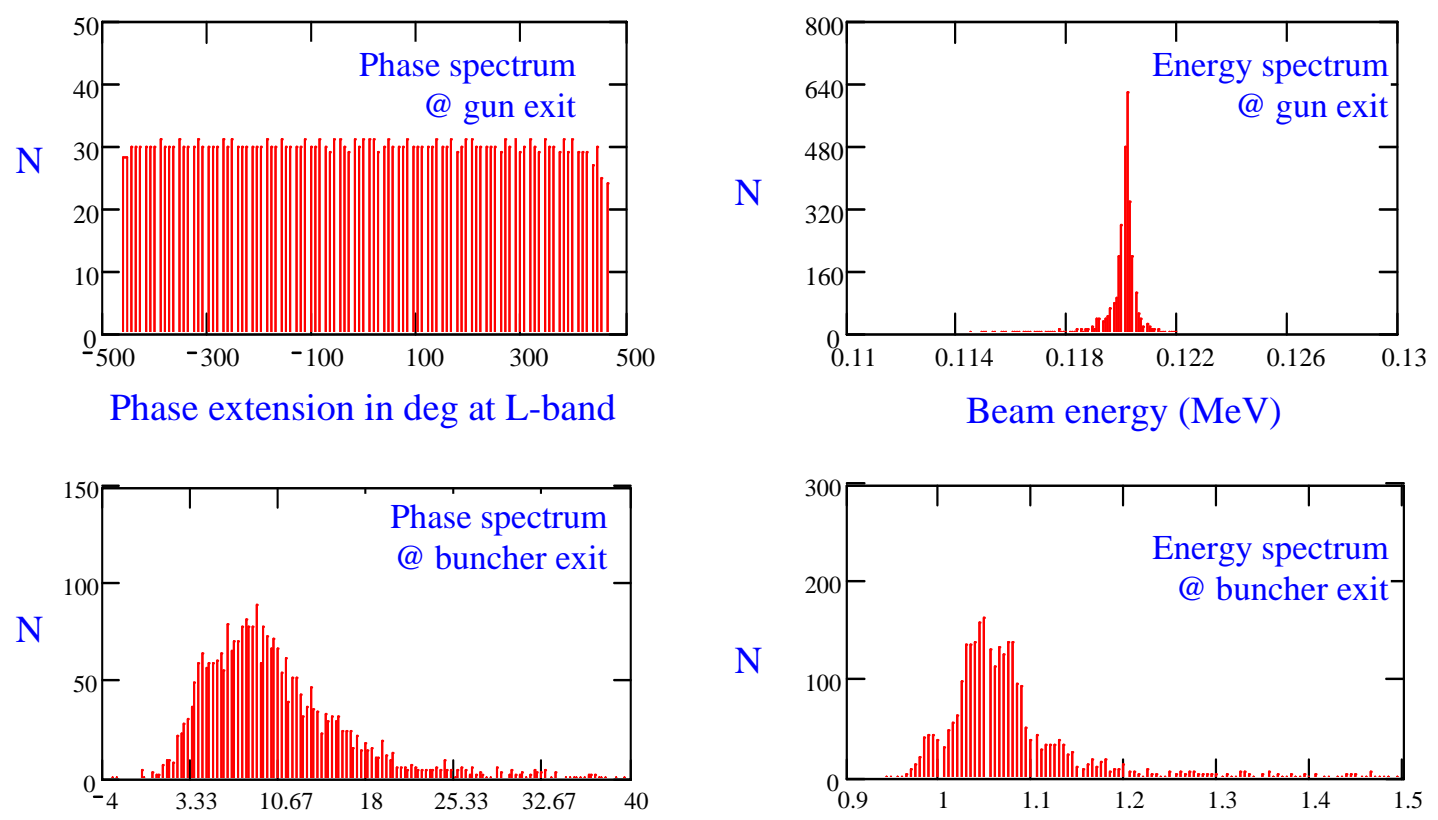

Phase extension in deg at L-band

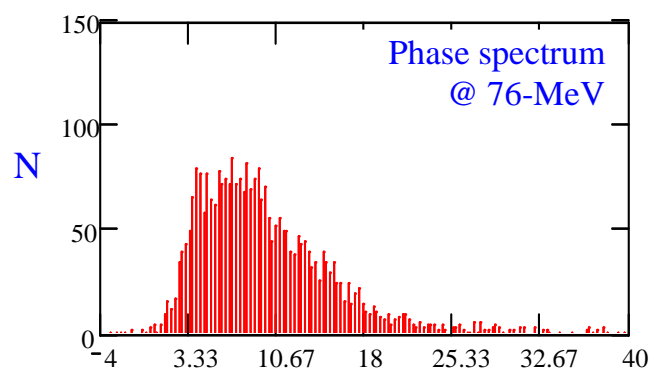

Phase extension in deg at L-band

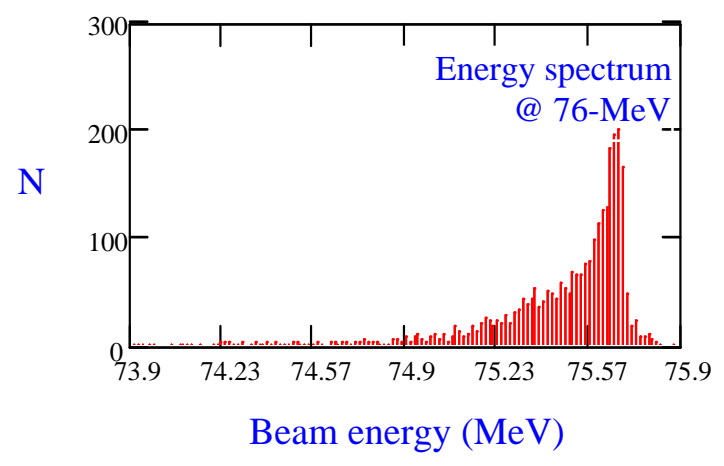

FIG. 4. Phase and energy spectra at the gun (top), buncher (middle), and $76 \mathrm{MeV}$ injector exit (bottom). 


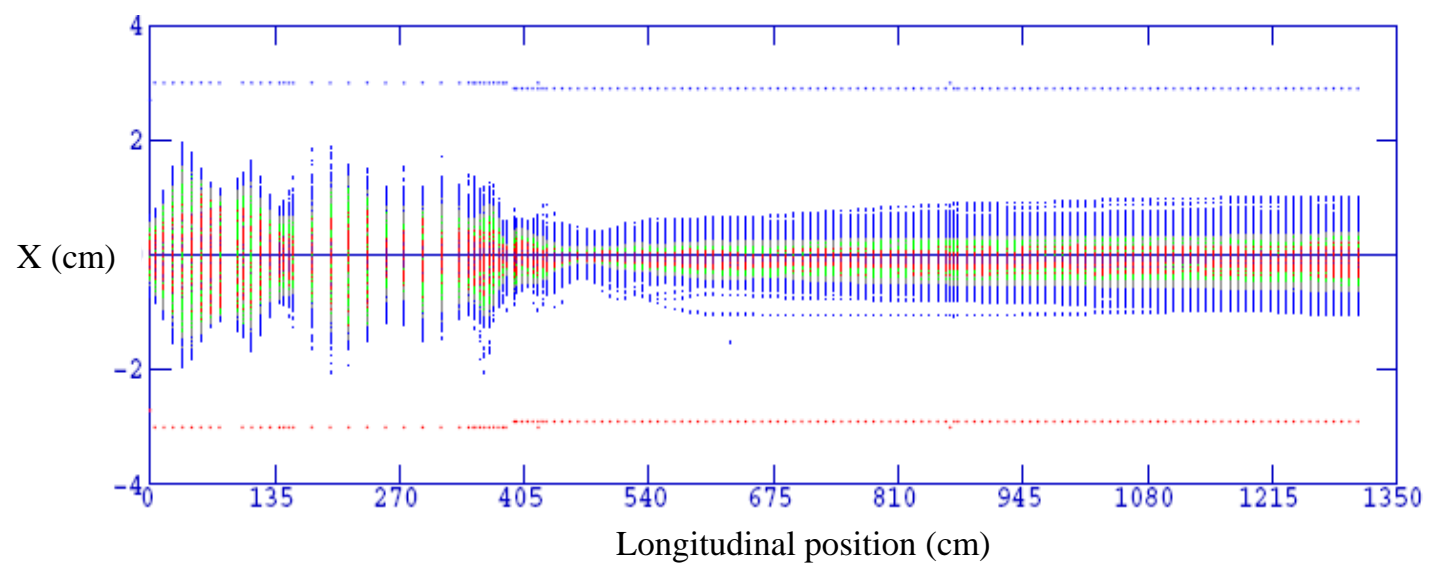

FIG. 5. Beam envelope along the 76-MeV injector.

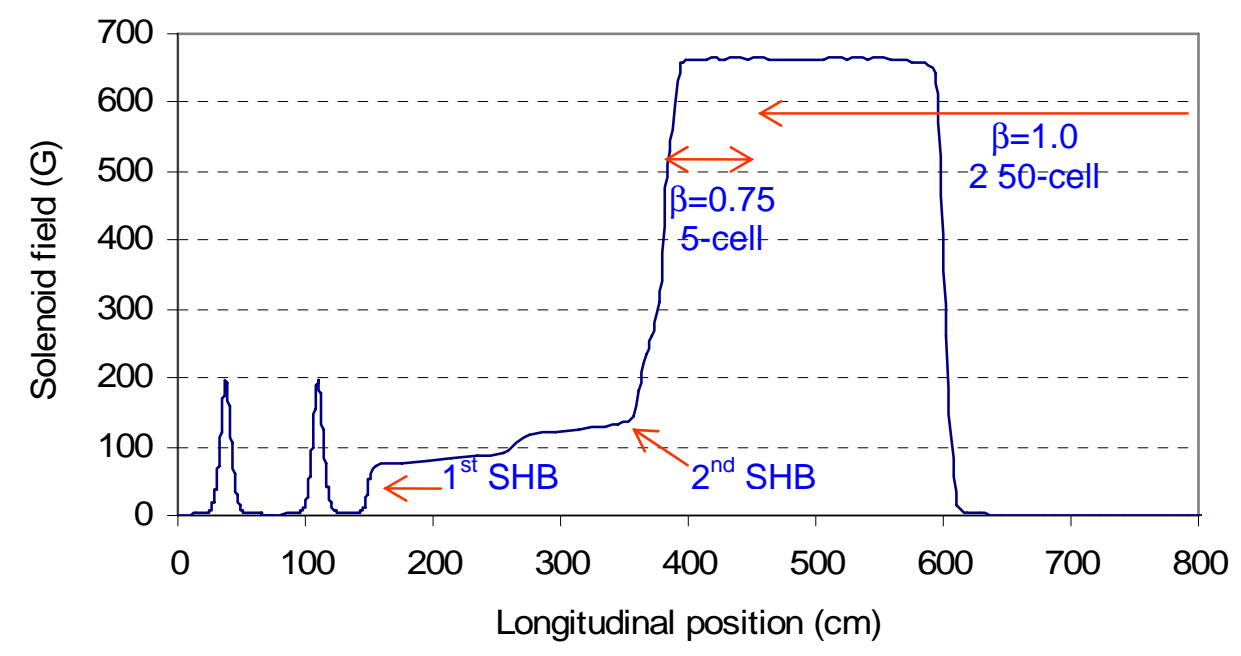

FIG. 6. Solenoid field map along the 76-MeV injector.

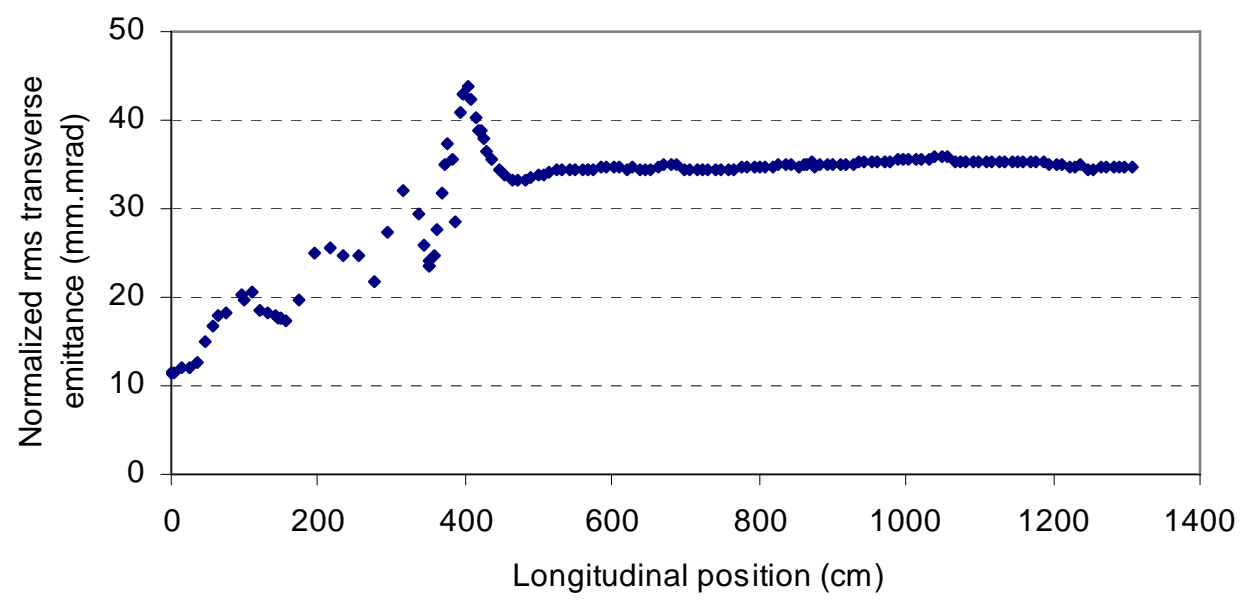

FIG. 7. Normalized rms emittance vs longitudinal position. 


\subsubsection{Comparisons between the $\beta=0.75 \mathrm{TW}$ and $\beta=1 \mathrm{SW}$ bunchers}

The beam performance is compared between this $\beta=0.75$ TW buncher and the $\beta=1$ SW buncher of Ref. [1]. Fig. 8 shows the phase and energy spectra at $76-\mathrm{MeV}$ for both bunchers. It shows that the TW buncher has a narrow phase extension and energy spread. Comparisons of 76-MeV beam performance are summarized in Table I, which explicitly indicates that all parameters in the TW buncher are better than in the SW one.

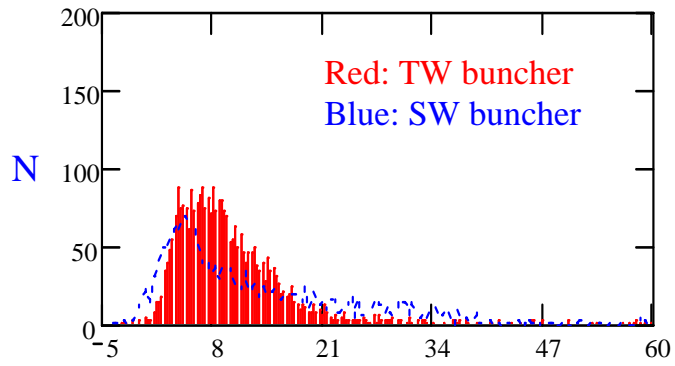

Phase extension in deg at L-band

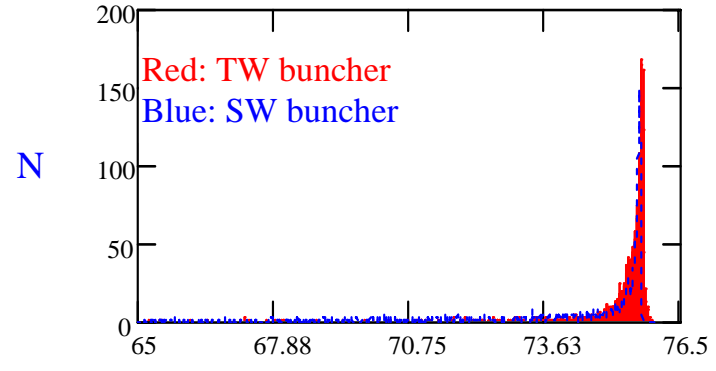

Beam energy $(\mathrm{MeV})$

FIG. 8. Phase and energy spectra at 76-MeV.

TABLE I. Comparisons of 76-MeV beam performance.

\begin{tabular}{|l|c|c|}
\hline & $\beta=0.75 \mathrm{TW}$ buncher & $\beta=1 \mathrm{SW}$ buncher \\
\hline Initial charge & $6.40 \mathrm{nC}$ & $6.40 \mathrm{nC}$ \\
Transmitted charge & $6.37 \mathrm{nC}$ & $6.13 \mathrm{nC}$ \\
Charge with a window & $5.88 \mathrm{nC}$ & $4.00 \mathrm{nC}$ \\
$(\Delta \mathrm{z} \times \Delta \mathrm{E}=21 \mathrm{deg} \times 1.2 \mathrm{MeV})$ & & \\
\hline Phase extension FWHM & 9 deg in L-band & 9 deg in L-band \\
Phase extension FW & $\sim 25 \mathrm{deg}$ in L-band & $\sim 50 \mathrm{deg}$ in L-band \\
\hline Energy spread FWHM & $<100 \mathrm{keV}$ & $<100 \mathrm{keV}$ \\
Energy spread FW & $<1.5 \mathrm{MeV}$ & $>3 \mathrm{MeV}$ \\
\hline Unnorm. edge emittance at gun exit & $70 \mu \mathrm{m}$ & $70 \mu \mathrm{m}$ \\
Norm. rms emittance at injector exit & $35 \mu \mathrm{m}$ & $50 \mu \mathrm{m}$ \\
\hline
\end{tabular}

\subsection{Vertical chicane, emittance station, and matching sections}

In the ILC electron source baseline design, horizontal doglegs are used to combine two normal conducting pre-acceleration systems and to clip off the electrons in the low energy tail before injecting into the SC electron booster linac. In September 2006, after the Vancouver Global Design Effort meeting, the backup normal conducting preacceleration system was removed to save the cost. But the energy collimation is still required. To make future services, repair and replacement particularly for cryomodules straightforward, a vertical chicane including several $90^{\circ}$ FODO cells inserted with bends 
is proposed. An energy collimator is installed near the maximum dispersion in the chicane. The tracking shows that the electrons in the lower end of the energy tail are efficiently collimated. A section to match Twiss parameters at the injector exit with the chicane is considered. The injector beam emittance is measured at a station downstream of the vertical chicane using conventional wire scanners. Two matching sections to match the chicane with the emittance station and the emittance station with the SC booster linac are needed. Figs. 9 and 10 show the optics and geometry in the region, respectively.

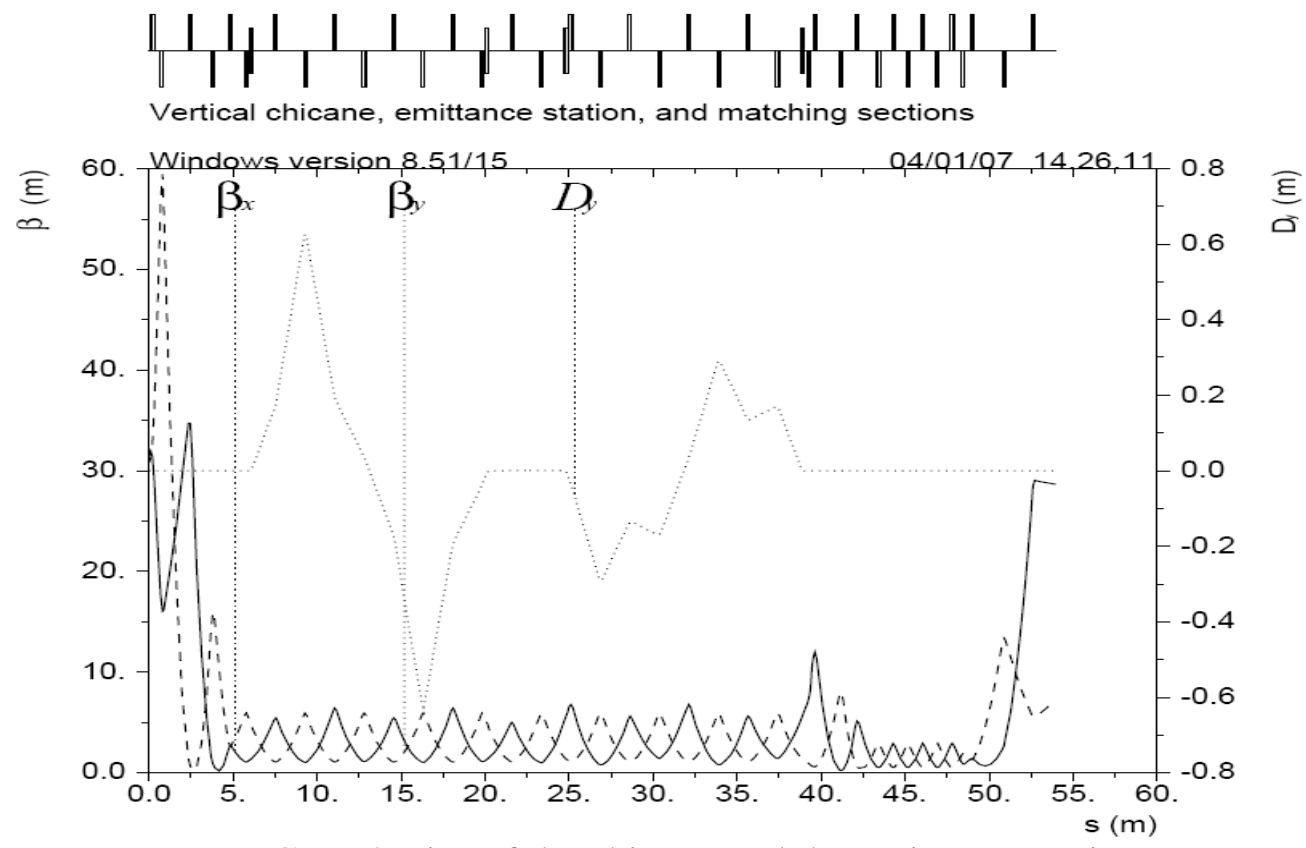

FIG. 9. Optics of the chicane and the emittance station.

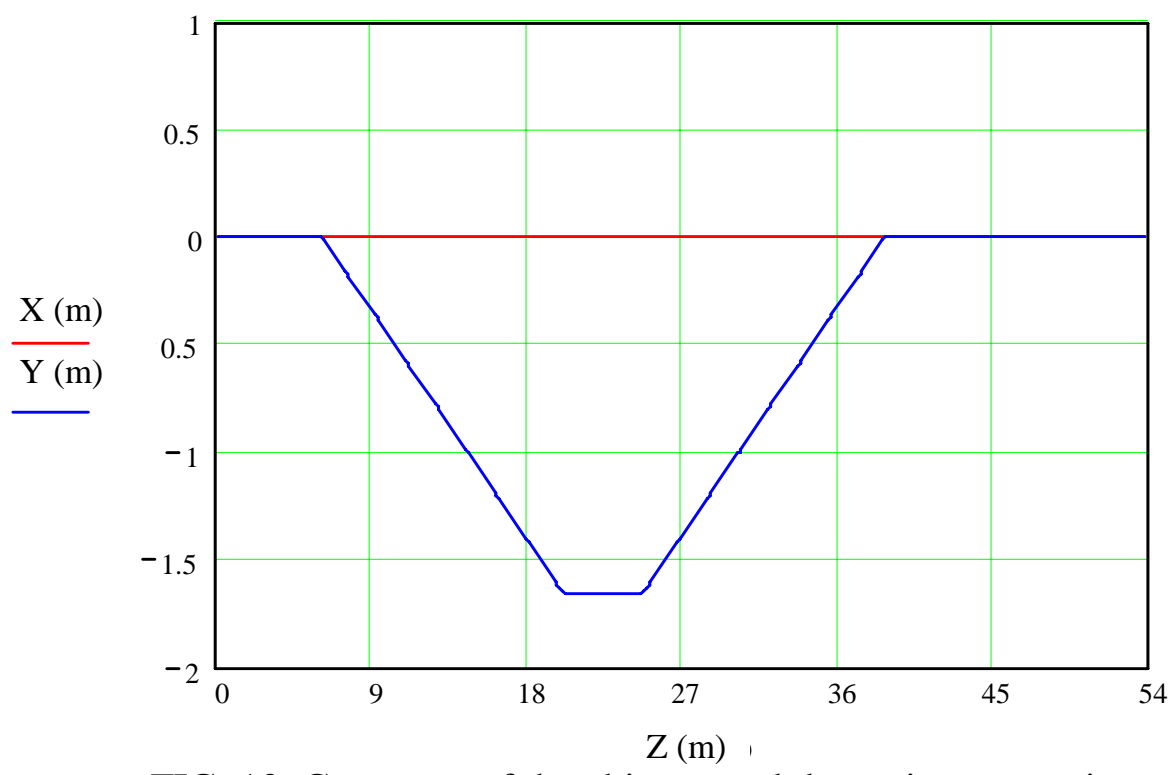

FIG. 10. Geometry of the chicane and the emittance station. 


\subsection{Optics of the booster linac system}

Twenty-one standard ILC-type SC cryomodules are used to accelerate the electron beam to $5 \mathrm{GeV}$. Typical FODO cells are used to focus the beam through the cryomodules. The booster linac has two sections. The energy in the first section ranges in between $76 \mathrm{MeV}$ and $1.717 \mathrm{GeV}$. One quadrupole sits inbetween two cryomodules for beam focusing, and the field strength of quadrupoles, $(\partial \mathrm{B} / \partial \mathrm{x}) \times \mathrm{L}$, is in the range of 0.10 $0.40 \mathrm{~T}$. In the second section, the electron beam is accelerated to $5 \mathrm{GeV}$. Its quadrupole spacing doubles that in the first section, and $(\partial \mathrm{B} / \partial \mathrm{x}) \times \mathrm{L}$ is in the range of $0.40-0.93 \mathrm{~T}$. The optics of the booster linac is shown in Fig. 11.

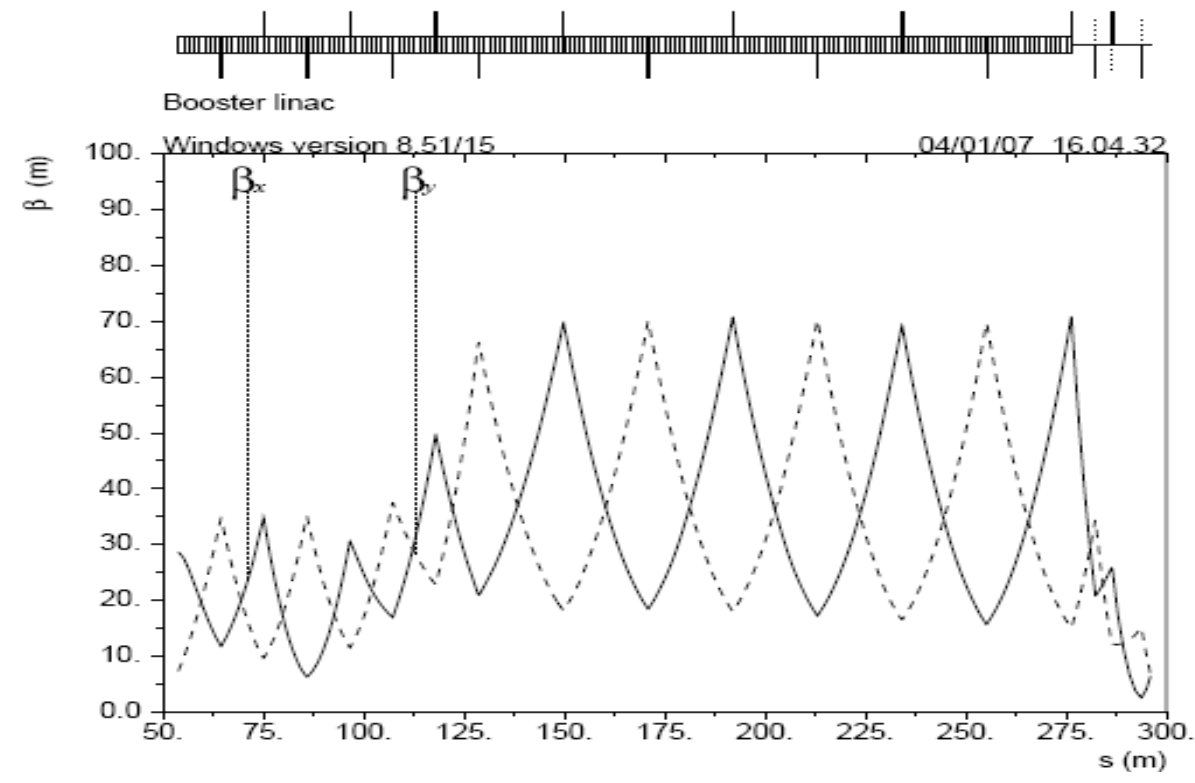

FIG. 11. Optics of the SC electron booster linac.

\subsection{Optics of the LTR system}

The electron Linac-to-Ring (LTR) system, which extracts the electrons from the booster linac system and injects them into the DR injection line, has two main functions: one is to rotate the electron spin vector to the vertical plane; and the other is to manipulate the energy compression to meet the DR longitudinal acceptance. The longitudinal polarization of the electrons is generated at the gun and preserved prior to the DR. In the DR, only electron spin directions parallel or anti-parallel to the magnet field - that is, transverse to the plane of the DR - will preserve their polarization. The LTR system consists of bending magnets and solenoid that change the spin of the electrons first from the longitudinal to the horizontal plane and then from horizontal to vertical, parallel to the magnetic field of the DR (the magnetic field in the DR is in the vertical plane). The spin precession with respect to the momentum vector caused by a bending angle, $\theta_{\text {bend }}$, is given by: 


$$
\theta_{\text {spin_bend }}=\frac{E(\mathrm{GeV})}{0.44065} \cdot \theta_{\text {bend }} \quad,
$$

where $E$ is the electron energy. Rotation of the spin vector in the horizontal plane by $n \cdot 90^{\circ}$ ( $n$ is an odd integer) from the longitudinal direction requires a total bending angle of $\theta_{\text {bend }}=n \cdot 7.929^{\circ}$ at $5 \mathrm{GeV}$. When an electron passes through a solenoid, its component of spin perpendicular to the solenoid field is rotated around the solenoid field axis. The rotation angle is approximately twice the rotation angle of the electron trajectory. The spin rotation angle caused by a solenoid is thus given by:

$$
\theta_{\text {spin__sole }} \approx \frac{B_{z} \cdot L_{\text {sole }}}{B \rho}
$$

where $B_{Z}$ is the longitudinal solenoid magnetic field, $L_{\text {sole }}$ is the solenoid length, $B \rho$ is the magnetic rigidity. For a $90^{\circ}$ of spin rotation from the horizontal to the vertical plane at 5 $\mathrm{GeV}$, a solenoid magnetic field integral of $B_{z} \times L_{\text {sole }}=26.23$ T.m is needed. An 8.3-mlong superconducting solenoid with $B_{z}=3.16 \mathrm{~T}$ is used.

The bunch decompression or energy compression can be realized by properly manipulating the linac RF phase with a suitable transfer function, $R_{56}=\int \frac{D_{x}}{\rho} d s$ ( $D_{x}$ is the dispersion, and $\rho$ is the bending radius), generated in bends:

$$
\Delta z \approx R_{56} \cdot \frac{d p}{p}+T_{566} \cdot\left(\frac{d p}{p}\right)^{2},
$$

where $\frac{d p}{p}$ is the induced correlated energy spread and $T_{566}$ is the second order term of transfer matrix. The first LTR arc having four FODO cells inserted with 8 bends is designed for the energy compression and the spin rotation. A bending angle of $7 \times 7.929^{\circ}=55.5^{\circ}$ is chosen in the design. The nominal $R_{56}$ is $86 \mathrm{~cm}$ but adjustable within the range of $\pm 30 \mathrm{~cm}$. After the bunch decompression, an RF voltage of $180 \mathrm{MV}$ provided by a 6-m-long superconducting linac is implemented to rotate the electrons in the longitudinal phase space to match the DR longitudinal acceptance.

The rest of the LTR system includes a section to have an additional $34.5^{\circ}$ horizontal bending, and a matching section with 4 quadrupoles and a double bend achromat used to match Twiss parameters at the DR injection line [4]. Its optics and geometry are shown in Figs. 12 and 13, respectively. 

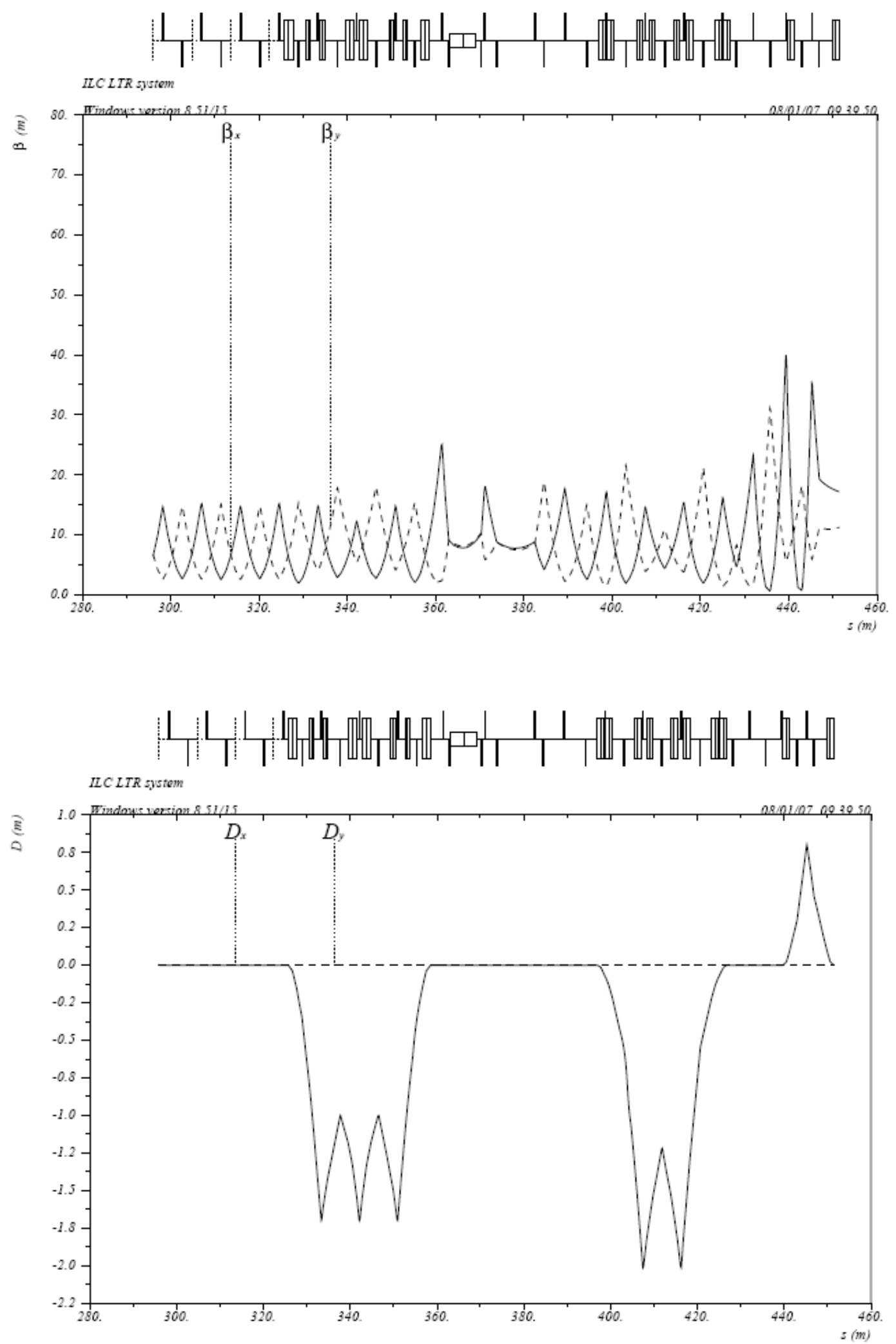

FIG. 12. Optics of the LTR system: $\beta$ functions (top), dispersion (bottom). 


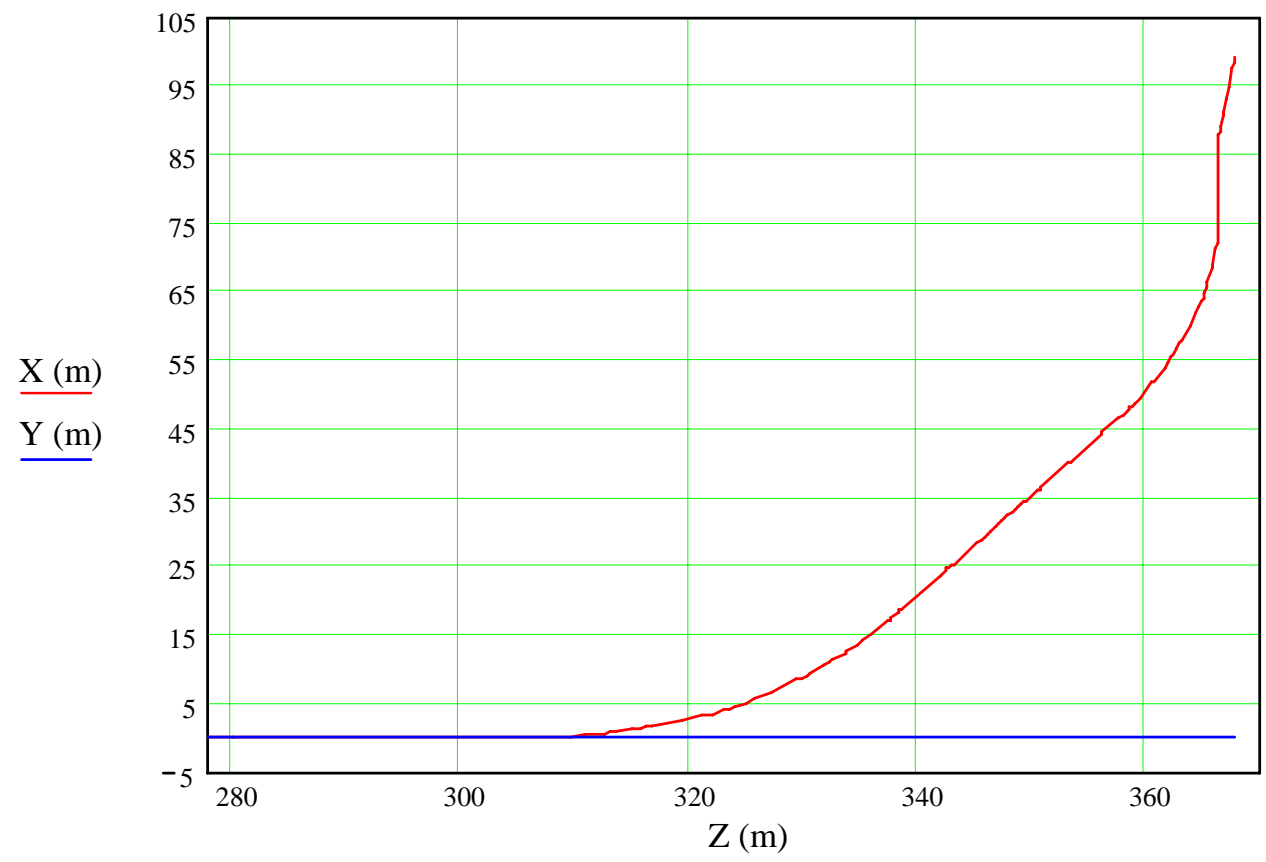

FIG. 13. Geometry of the LTR system.

\subsection{Emittance measurement, PPS stoppers, and 5-GeV beam dump}

The 5-GeV beam emittance is measured using four laser wire scanners located the standard FODO cells immediately downstream of the booster linac. Three Personal Protection System (PPS) stoppers with 1-m in each are installed in the drifts in between the bends and quadrupoles of the first LTR arc. A 5-GeV beam dump is located downstream of the emittance measurement station. To dump a 5-GeV beam, the first bend in the LTR needs to be turned off. The dump line, shown in Fig. 14, includes a defocusing quadrupole, a dump bend, a 0.8-m long focusing quadrupole, and a 9.1-m long drift to the dump window. At the dump window, for $\pm 0.1 \%$ and $\pm 10 \%$ of energy spread, the half edge beam sizes $\sigma x / \sigma y$ are $0.52 \mathrm{~cm} / 1.1 \mathrm{~cm}$ and $13.7 \mathrm{~cm} / 1.1 \mathrm{~cm}$, respectively, which meet the dump window specifications [5]. At the monitor location shown in Fig. 14, the dispersion dominates the beam size, and thus the dump line can be used as an energy spectrometer with $0.1 \%$ of resolution. 


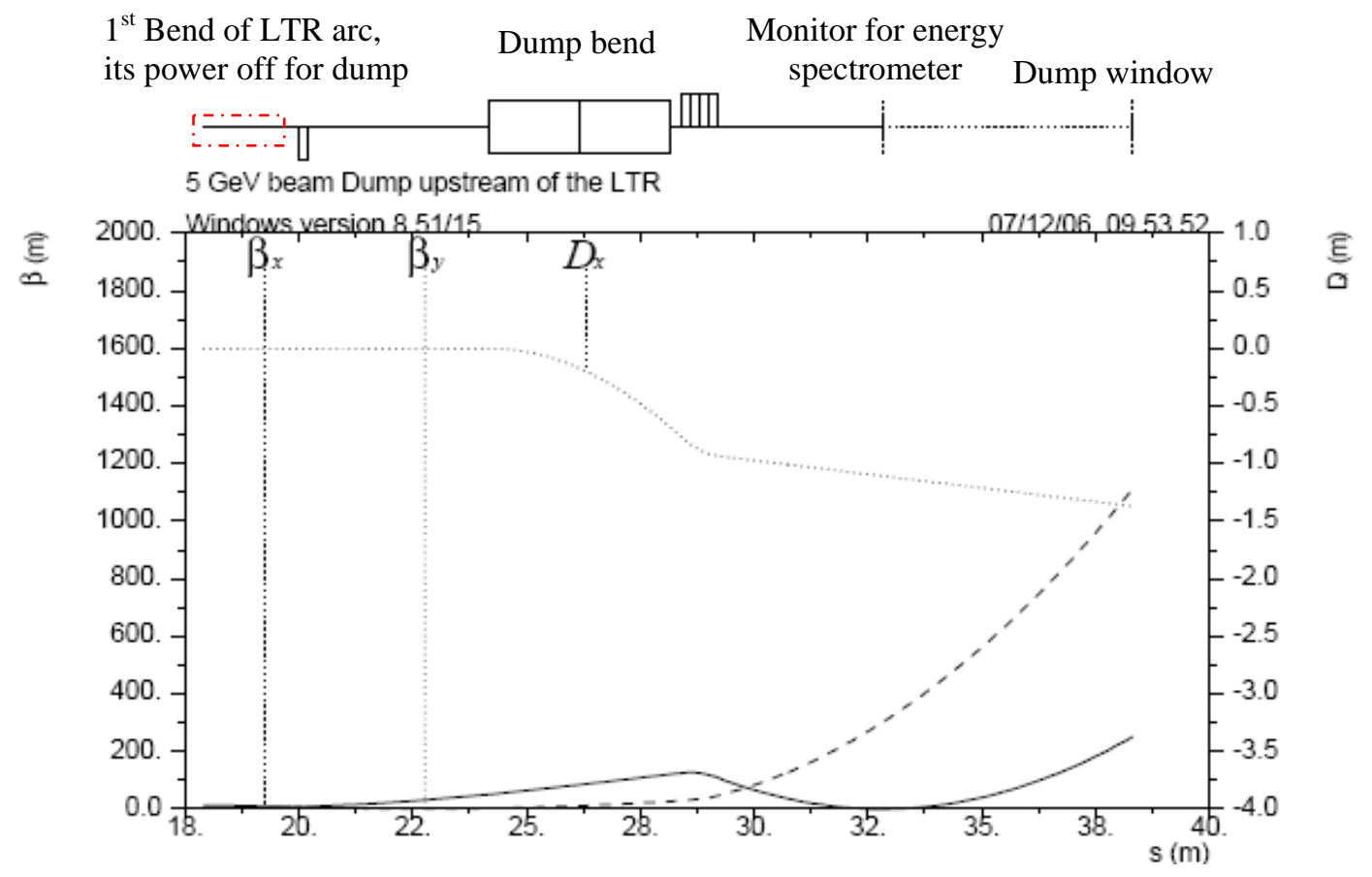

FIG. 14. 5-GeV beam dump line downstream of the booster linac system.

\section{Multi-particle tracking from the $120 \mathrm{kV}$ DC-gun to the entrance of the DR injection line}

\subsection{Tracking without magnet errors}

Multi-particle tracking from the gun to the entrance of the DR injection line has been performed. PARMELA is first used to track electrons from the gun to the $76-\mathrm{MeV}$ normal conducting pre-accelerator. The ELEGANT code [6] is then used to track the beam through the rest of electron beamline including the vertical chicane, the SC booster linac, and finally the LTR system. Electron 6-D coordinates at the pre-accelerator exit from the PARMELA simulation are used as the input data in the ELEGANT code tracking. Electrons in the low energy tail from the 76-MeV injector are collimated with an energy collimator installed near the maximum dispersion in the vertical chicane; its length is $10 \mathrm{~cm}$, and the half apertures in the $\mathrm{x}$ - and $\mathrm{y}$-plane are $1.5 \mathrm{~cm}$ and $1.2 \mathrm{~cm}$, respectively. To accommodate more electrons within the DR 6-D acceptance $A_{x}+A_{y} \leq 0.09 \mathrm{~m}$ and $\Delta E \times \Delta z \leq( \pm 25 \mathrm{MeV}) \times( \pm 3.46 \mathrm{~cm})$ [7], the energy compression is optimized in the LTR before injecting into the DR injection line. For that purpose, the booster linac upstream of the LTR runs the booster linac RF phase off-crest to create a suitable correlated energy spread. Fig. 15 shows the longitudinal phase space at the LTR exit after energy compression. Tracking shows that $94 \%$ of the electrons from the gun can survive the transport through the complete beamline based on the physical apertures of the beam pipes listed in Table II. Next, we need to determine whether these survived 
electrons are captured within the DR 6-D acceptance. To calculate electrons capture efficiency within the DR 6-D acceptance, first we calculate the Twiss parameters $\alpha, \beta$, and $\gamma$ from the beam distribution. In the x-plane, these parameters are given by:

$$
\alpha_{x}=-\frac{\left\langle x \cdot x^{\prime}\right\rangle}{\varepsilon_{x}}, \beta_{x}=\frac{\left\langle x^{2}\right\rangle}{\varepsilon_{x}} \text {, and } \gamma_{x}=\frac{\left(1+\alpha_{x}^{2}\right)}{\beta_{x}} \text {, }
$$

where the un-normalized rms emittance is $\varepsilon_{x}=\sqrt{\left\langle x^{2}\right\rangle\left\langle x^{\prime 2}\right\rangle-\left\langle x x^{\prime}\right\rangle^{2}}$. Parameter calculations for $\alpha_{y}, \beta_{y}$, and $\gamma_{y}$ in the y-plane are analogous to the x-plane. Twiss parameters $\alpha_{x}\left(\alpha_{y}\right), \beta_{x}\left(\beta_{y}\right)$, and $\gamma_{x}\left(\gamma_{y}\right)$ define a family of ellipses with the same ratio of semi-axes. For particles with index $i$, its un-normalized emittance is

$$
\varepsilon_{x, i}=\gamma_{x} \cdot x_{i}^{2}+2 \alpha_{x} \cdot x_{i} \cdot x_{i}^{\prime}+\beta_{x} \cdot x_{i}^{\prime 2}
$$

and the $\varepsilon_{y, i}$ is calculated analogously. Given the normalized transverse emittance, $\gamma_{i, \text { energy }} \cdot\left(\varepsilon_{x, i}+\varepsilon_{y, i}\right) \leq 0.09 \mathrm{~m}$, where $\gamma_{i, \text { energy }}$ is the Lorentz factor for the particles with index $i$, and the longitudinal phase space is within the DR acceptance, the particle is considered captured. Without the LTR, the tracking shows that $88 \%$ of electrons from the gun are captured within the DR 6-D acceptance. In other words, $6 \%$ of the electrons are beyond the DR acceptance without energy compression. With the LTR energy compression, the complete tracking shows that all surviving electrons $-94 \%$ of electrons from the gun - are captured within the DR 6-D acceptance at the entrance of the DR injection line.

\subsection{Tracking with magnets error and orbit corrections}

Magnet field errors and other machine imperfections, such as misalignments which can be expressed equivalent to field errors, are randomly spread along the beamline. These errors can perturb beam orbits and beam size, and may affect beam performance. Tracking with the typical magnet errors listed in Table III shows that the number of captured electrons within the DR acceptance does not change even without an orbit correction. However, the orbit perturbation may eat up the physical apertures, and thus the perturbed orbit needs to be corrected. The correctors should be positioned near the maximum values of the $\beta$-function in the corresponding plane to efficiently correct the orbit. In other words, one horizontal corrector is placed near a focusing quadrupole while one vertical corrector near a defocusing quadrupole. After the orbit correction, all electrons are captured within the 6-D acceptance. We tested different random seeds of magnet errors, and the results are similar. Fig. 16 shows the transverse phase spaces at the entrance of the DR injection line without errors, with errors but without orbit correction, and with errors and orbit correction. It shows that the orbit is properly corrected. 


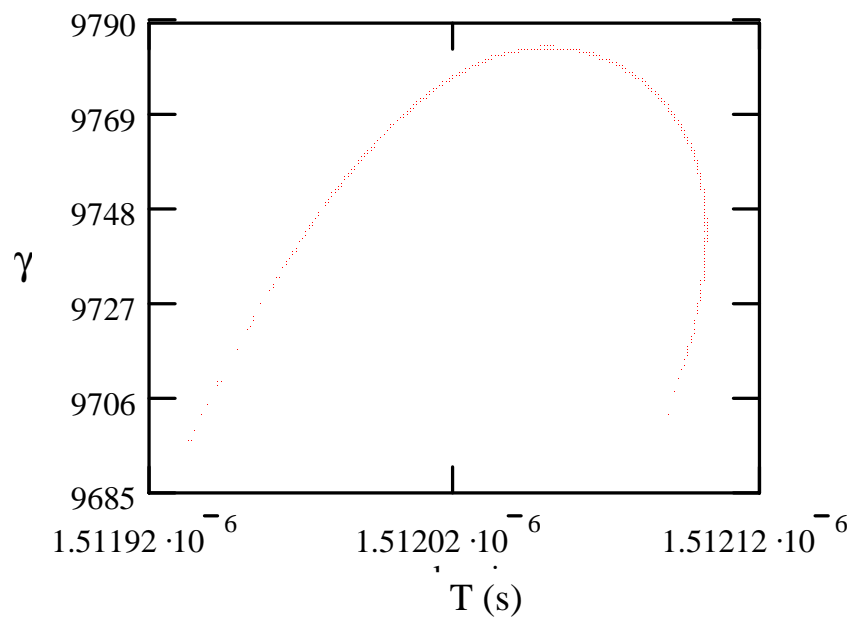

FIG. 15. Longitudinal phase space at the entrance of the DR injection line after the energy compression; all electrons are within the DR 6-D acceptance; $\gamma$ is the Lorentz factor.

TABLE II. Preliminary definition for physical apertures along the beamline.

\begin{tabular}{|c|c|}
\hline Components & Half aperture in $\mathrm{x} / \mathrm{y}(\mathrm{cm})$ \\
\hline Bunching system & $2.5 / 2.5$ \\
SHBs & $2.5 / 2.5$ \\
L-band buncher & $2.0 / 2.0$ \\
Pre-Accelerator & $1.5 / 1.5$ \\
\hline Chicane & $1.5 / 1.5$ \\
Emittance station & $3.7 / 3.7$ \\
\hline Booster linac & \\
\hline LTR & $3.7 / 3.7$ \\
RF section & $2.0 / 2.0$ \\
Solenoid & $5.5 / 3.0$ \\
Others & \\
\hline
\end{tabular}


TABLE III. RMS values of magnet errors used for tracking.

\begin{tabular}{|l|c|c|c|}
\hline & $\begin{array}{c}\text { Misalignment in } \mathrm{x} \\
\text { and y plane }\end{array}$ & Field error & Rotation error \\
\hline Quadrupole & $\begin{array}{c}\Delta \mathrm{x}=200 \mu \mathrm{m} \\
\Delta \mathrm{y}=200 \mu \mathrm{m}\end{array}$ & $0.1 \%$ & \\
\hline Sextupole & $\begin{array}{c}\Delta \mathrm{x}=200 \mu \mathrm{m} \\
\Delta \mathrm{y}=200 \mu \mathrm{m}\end{array}$ & $0.1 \%$ & \\
\hline Bend & $\Delta \mathrm{x}=200 \mu \mathrm{m}$ & $0.1 \%$ & $0.3 \mathrm{mrad}$ \\
& $\Delta \mathrm{y}=200 \mu \mathrm{m}$ & & \\
\hline
\end{tabular}
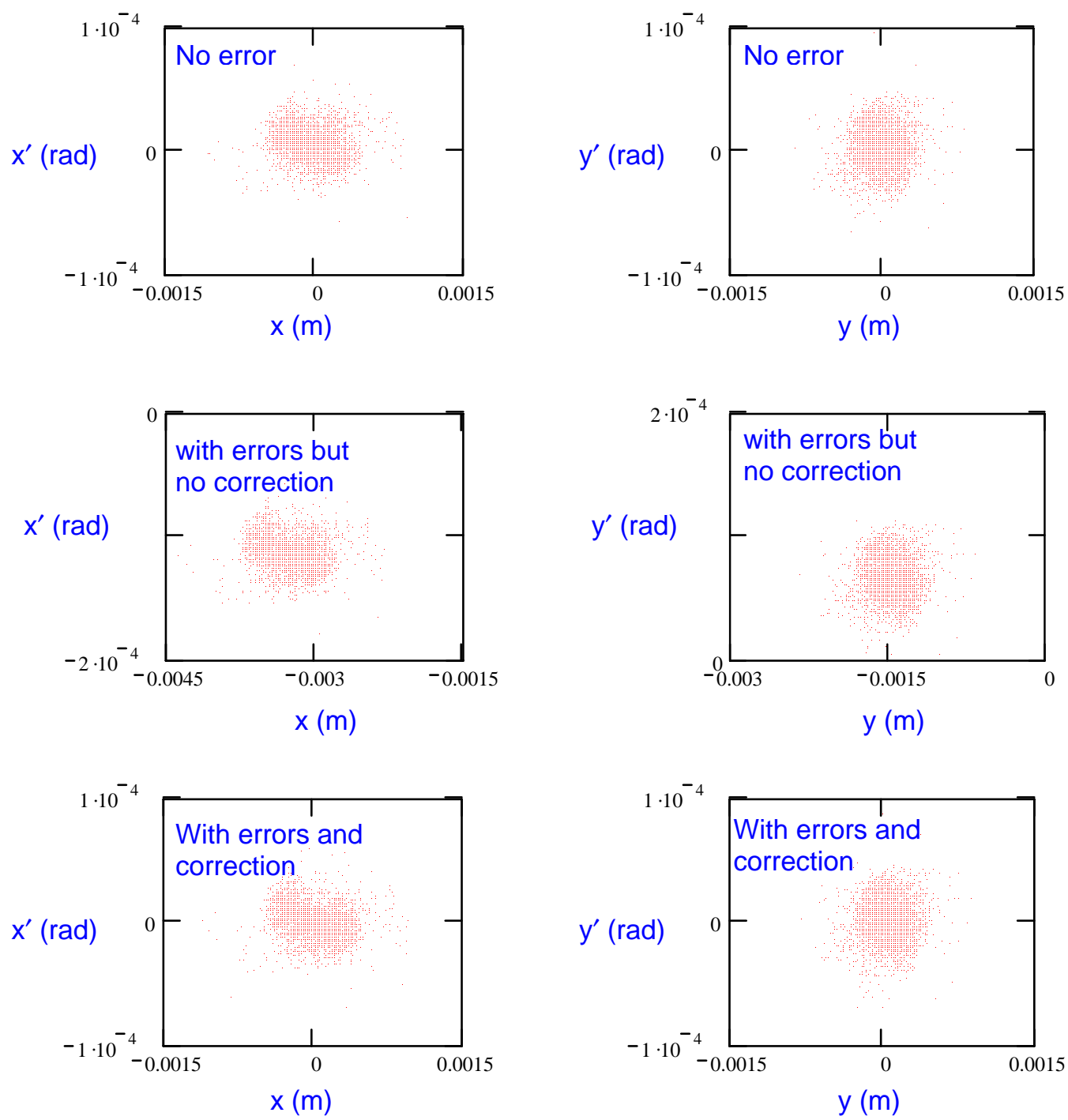

FIG. 16. Transverse phase spaces at the entrance of the DR injection line without magnet errors, with errors but without correction, and with errors and correction. 


\section{Summary and future work}

The bunching system and transport optics for the 120-kV DC-gun based ILC polarized electron source are developed; start-to-end particles tracking with and without magnet errors is performed. The tracking shows that $94 \%$ of electrons from the gun are captured within the DR 6-D acceptance at the entrance of the DR injection line. Orbit perturbations caused by magnet errors along the beam line are corrected.

Looking toward the Engineering Design Report, a lot more detail work is needed including: (1) bunching system optimizations to meet the engineering design; (2) designing injector for higher gun voltage (e.g., $200 \mathrm{kV}$ ), which may allow shorter initial micro bunch length (e.g., 700 ps); (3) beamline optics and physical aperture optimizations to meet the engineering design; (4) component tolerances definition; and (5) defining tuning requirements.

\section{Acknowledgement}

Feng Zhou thanks Drs. Nan Phinney and Tor Raubenheimer for the encouragements. The work is supported by DOE contract DE-AC02-76SF00515.

\section{References}

[1] A. Curtoni and M. Jablonka, TESLA 2001-22.

[2] J. Wang, et al., "Studies of room temperature accelerator structures for the ILC positron source”, PAC’05, Knoxville, 2005.

[3] L. Young, PARMELA manual, LA-UR-96-1835, 2001.

[4] I. Reichel, Private communication, 2006.

[5] D. Walz, $2^{\text {nd }}$ ILC accelerator workshop, Snowmass, Aug. 14-27, 2005.

[6] M. Borland, Technical Report No. LS-287, ANL, 2000.

[7] A. Wolski, private communication, Nov. 2006. 